\title{
Development of a modified open set sentence test for assessment of speech perception skills in Hindi preschool children
}

\begin{abstract}
Introduction: Clinical assessment of speech perception skills is very important for several reasons. However, it is routinely assessed in older children and adults but not in very young children mainly due to lack of appropriate tools, especially in Indian languages. Considering the limitations of closed-set and open-set tests for young children modified open set test would be appropriate.
\end{abstract}

Aim: To develop a modified open set sentence test in Hindi based on the Mr. Potato Head Test framework using appropriate toy and sentence material and employ the developed test to assess children with normal hearing in the age range of 18 to 48 months.

Method: Two sentence lists with ten sentences each and one practice list with five sentences were developed. The test was recorded in a professional studio by a sound engineer and sentences were calibrated using the Bruel \& Kjaer-Type-2250 sound level meter (SLM) so that the test could be administered via speakers using a laptop. The test was administered in a quiet room, directly through a laptop connected to speakers on 200 Hindi speaking children with normal motor and speech developmental milestones and bilateral pass result on OAE screening.

Results and discussion: There was a significant difference in the scores between the 5 age groups of children with normal hearing. There was an increase in scores as age increased, thus indicating improved performance with increasing age. There was also no significant difference in the performance on List 1 and List 2 for all age groups indicating that the two lists were equivalent. Item reliability was also observed to be high.

Conclusion: The developed Modified Open Set Sentence Test can be successfully used to assess speech perception skills in young children with normal hearing. This test has a potential to be a useful clinical tool when used as a part of a test battery and also play a role in monitoring the progress of an intervention program.

Keywords: speech perception, modified open set, Hindi, preschool children, sentence test
Volume II Issue 3 - 2019

\author{
Yaashna Rajani MASLP, ' Aparna Nandurkar \\ $\mathrm{PhD}^{2}$ \\ 'Practicing Audiologist and Speech-Language Pathologist, \\ Mumbai \\ ${ }^{2}$ Ali Yavar Jung National Institute of Speech and Hearing \\ Disabilities, Mumbai
}

Correspondence: Aparna Nandurkar, Lecturer (Speech and Hearing), Department of Audiology, AYJNISHD, Bandra Reclamation, KC Road, Bandra West. Mumbai 400050. India, Tel +919820446304;

Email aparnanitinnandurkar@gmail.com

Received: April 05, 2019 | Published: May 06, 2019

\section{Introduction}

The term speech perception refers to the process by which the motoric/ acoustic patterns of speech become linguistic structures in listener's mind. ${ }^{1}$ Speech perception is a complex process that allows us to concentrate on our goals of making sense out of speech. ${ }^{2}$ Due to this ability of speech perception, humans acquire language which makes them unique as they can communicate using structured codes of a language. Speech perception assessment when undertaken clinically for persons with hearing loss, serves various purposes. It helps to classify the degree and type of hearing loss by cross checking the results obtained through other tests from the audiological test battery and reflects the degree of communication impairment created by the hearing loss. Such testing provides a measure of how well listeners understand speech in a controlled environment roughly reflecting their performance in everyday listening situations, ${ }^{3,4}$ and provides valuable information for planning and managing auditory (re)habilitation. A quantitative speech perception measure helps in predicting the effectiveness of a particular medical or surgical treatment and sensory device. ${ }^{5-9}$ It helps in monitoring a child's performance throughout the therapeutic process and measuring the success of an intervention program. Speech perception assessment is used in various forms of research to monitor participants' performance in research paradigms.

The testing of speech perception abilities in young children, especially those with hearing loss, is a considerable challenge. Difficulties are encountered owing to various factors such as:Vocabulary of very young congenitally deaf children is minimal. They are unable to make use of auditory speech memory, since this is absent in most cases. Their cognitive immaturity must also be taken into account. ${ }^{10,11}$ The ability to cooperate with the tester over an extended period is also limited, since young children tire more easily, with a corresponding decline in attentiveness and concentration.

There are various stimuli which can be used for speech perception assessment and these include vowels, consonants, nonsense syllables, words, minimal pairs, sentences, connected speech, etc. Nonsense Syllable tests measure speech perception ability more accurately as compared to meaningful words as they minimize the contextual cues provided from within the stimuli. Phoneme recognition tests are usually closed set tests and provide a descriptive profile of listeners' 
phonemic errors while perceiving speech. Meaningful Monosyllabic tests are phonetically and/ or phonemically balanced monosyllabic lists that are used very frequently in clinical audiology. ${ }^{12-14}$ Though these tests are used frequently, the kind of diagnostic information gained from them is limited. Sentence tests are considered to provide a measure of an individual's performance in everyday listening situations as they are similar to realistic situations where the stimuli contain considerable amount of redundancy and contextual cues. But the sentence tests are criticized for not providing analytical perception abilities as there is full scope of performing closure for responding correctly. Some researchers tried to develop tests to overcome these drawbacks. Speech Perception in Noise (SPIN) test ${ }^{15}$ tried to overcome this by having sentences with low and high probability. Cox, Alexander and Gilmore ${ }^{16}$ developed a different approach of testing sentence perception with the help of the Connected Speech Test (CST). The test consists of a variety of passages of conversationally produced connected speech and the scoring is done based on the number of key words perceived correctly. There are some tests that use stimuli different from the above-mentioned options, e.g. Auditory Numbers Test ${ }^{17}$ uses number words one through five via Monitored Live Voice with a closed set response task, while the Sound Effect Recognition Test (SERT) ${ }^{18}$ uses familiar environmental sounds as an alternative to speech stimuli for assessing auditory discrimination abilities of children not capable of differentiating verbal stimuli.

Closed set tests limit the number of responses and thus the number of alternatives influence the guess rate. Since there is a limited number of options available to choose from, it can inflate performance and it is important to recognise that. The closed set testing format has remained popular in clinical settings because it can be simply and rapidly administered and scored. ${ }^{19}$ The results obtained from closed set tests however do not represent how the child would perform in everyday situations. Closed set tests are commonly used when the child is unable to perform an open set test. Open set protocols provide unlimited number of stimulus alternatives and therefore are more challenging, but the results are more representative of what the child is likely to encounter in everyday situations. However, use of open set tests in children is challenging.

\section{Need for the study}

Assessment of speech perception is a cornerstone of clinical audiological assessment and has further taken a boost after the advent of cochlear implantation, especially in young children. In 2000, FDA guidelines approved cochlear implantation (CI) for children as young as 1 year of age..$^{20}$ Moreover, some surgeons are providing CIs to infants under 1 year of age when there is availability of clear evidence that they are not receiving benefits from conventional hearing aids or in children with family history of congenital deafness or history of meningitis who are at risk of cochlear ossification. In Indian scenario, there are Government programs funding CIs at large and this increases the population of young cochlear implanted children considerably. For example, Government of Kerala under the Sruthitharangam scheme ${ }^{21}$ is providing free surgery to children between 0-3 years. The ADIP Scheme of Government of India ${ }^{22}$ is providing free implantation for children with pre-lingual hearing impairment below 5 years of age. Further, the population of early-implanted deaf infants is likely to increase substantially because of broadening of candidacy criteria and because hearing loss can now be detected at younger ages due to effective screening programs. For young children there is more focus on indirect testing of speech perception through parental reports such as IT-MAIS, ${ }^{23}$ MAIS,,${ }^{24}$ and PEACH,,${ }^{25}$ word level testing (ESP, WIPI) ${ }^{26,27}$ and Ling 6-sound test. ${ }^{28}$ It must be kept in mind that in general we speak in discourse and not in words or syllables. Use of sentence stimuli for testing speech perception is appropriate for validly sampling everyday conversational speech as they contain some amount of redundancy and contextual cues.

In order to overcome difficulties encountered in testing very young children, it is necessary for the clinician to deal sensitively with the child and use appropriate testing procedures. Open set tests cannot be used with all children as they might be unable to give verbal responses and closed set tests may not adequately represent a listener's performance in natural situations and hence a modified open set test is a good option with young children. Considering the importance of clinical speech perception assessment in young children in general and cochlear implanted children in particular, it is the need of the hour to develop a test which enables us to assess speech perception in young children; a modified open set test will suit this requirement adequately.

There is also lack of information about speech perception abilities of young normal hearing Hindi speaking children and this information is necessary to make conclusions about outcomes with cochlear implants in young children. Hindi is the native language of approximately $41.03 \%$ of Indians ${ }^{29}$ i.e. there are 422,048,642 speakers using Hindi as first language. It is spoken as a second or third language by over 300 million Indians. Despite the pressing need for Hindi material for speech perception testing, there is a scarcity of Hindi test material in India, especially for young children.

Currently there are only few published studies which report sentence recognition/speech perception abilities in Hindi speaking children with hearing impairment, but none on normal hearing Hindi pre-schoolers. Further, most of them have been done as a part of some projects or dissertations and none of the tests/stimulus materials have been standardized, published or are commercially available. Hence this study was taken up to develop a modified open set sentence test for assessing speech perception in young children, based on the Mr. Potato Head ${ }^{30}$ framework.

\section{Aim}

To develop a modified open set sentence test in Hindi based on the Mr. Potato Head Test ${ }^{30}$ framework using appropriate toy and sentence material and employ the developed test to assess children with normal hearing in the age range of 18 to 48 months.

\section{Methodology}

The methods are described under two phases: 1) Test development and 2) Testing of normal hearing Hindi pre-schoolers.

\section{Phase I - test development}

The main aim was to develop a modified open set sentence test which requires manipulation of objects as a response and which can be administered through a laptop with external speakers in a quiet setting. The following were the steps involved in test development:

Selection of the toy: Before constructing the sentences, selection of the appropriate toy was done. For this purpose, a survey was conducted among 40 parents, 40 teachers and 20 children of the mentioned age group to find out which are the five most popular toys among these children. It was observed that the cartoon character 
Chhota Bheem (Chhota meaning 'small' and Bheem is the name of a boy) was the most popular and known to all children. The character is also commonly depicted in stories and is available as a soft toy and different types of merchandise for children. Hence, this toy was chosen for the test. The size of the toy ( 23 inches) was such that it was not too small to perform the activities required by the stimulus sentences, and not too large to be handled by the young children. The other material and props were chosen accordingly after the development of the test sentences.

Development of stimulus sentences: To ensure that the vocabulary and syntax of the stimulus sentences is appropriate for the target age group, the sentences were made using vocabulary from the preprimary level Hindi text books and activity books and ensuring that they are used in daily communication situations. While constructing the sentences an attempt was made to include nouns (household objects, common animals, fruits, and vegetables), verbs, adjectives, and few prepositions and the Mean Length of Utterance (MLU) of sentences increased as the list progressed. To begin with, a pool of 50 sentences was prepared depicting various actions/activities that children of this age can perform with the toy. The sentences from the Mr. Potato Head Test were used as the guideline.

Appropriateness of test material: To assess the appropriateness of the material the sentences were given to parents and teachers of normal hearing children within the age range of 18 months to 48 months for rating the familiarity of the sentences on a three-point rating scale (1-Very familiar, 2-Familiar, 3-Not familiar). The sentences that were rated as familiar by $100 \%$ of the respondents were selected in the final list. The lists were also validated by one audiologist and three special educators teaching children with hearing impairment. After this process of validation, a total of thirty-five sentences was selected, which were divided into two lists of fifteen sentences each and one practice list of five sentences.

Recording of the stimuli: To decide the speaker for recording the test material, the five sentences from the practice list were recorded as spoken by five proficient Hindi speaking female speakers using a Digital Sony voice recorder in a quiet room. These samples were given to four ASLPs for rating the speaker, wherein they were asked to give a five-point rating where ' 1 ' is worst and ' 5 ' is the best. The ratings were based on voice projection, diction, quality and clarity. Based on the ratings of the four ASLPs, a twenty-four-year-old native Hindi female speaker was selected for recording the stimulus sentences. Prior to the recording, the speaker was instructed to read out the material using least possible intonation, equal stress and uniform loudness (monotonous). To create digitised pre-recorded sentence stimuli, the sentence stimuli were recorded in a professional recording studio by a sound engineer.

Calibration of test setup: The stimulus material was to be presented at conversational level directly through the laptop in a quiet environment. To calibrate the presentation level, the recorded stimuli were played via a HP laptop connected to Mosto 2.0 loudspeakers placed at $45^{\circ}$ azimuth. The microphone of a Bruel \& Kjaer-Type-2250 sound level meter (SLM) was held at $1 \mathrm{~m}$ from the speakers and measurements were made as the stimuli were played from the laptop. The SLM was set on "fast" mode. The volume of the speaker was manipulated till readings between 55-60 dB SPL were consistently obtained on the SLM. The volume setting on the speaker which resulted in this intensity was marked to enable consistent setting during test presentation. Also, an application on the iPad called Sound Meter was used during this measurement session and before seating each participant, to ensure that the correct distance from the speaker was maintained.

Pilot Study: A pilot study was conducted to ensure that the stimuli prepared were appropriate for 18 to 48-month-old children, the children could follow the instructions and could carry out the task of manipulating the toy as required. This was done on 10 normal hearing children - two from each sub group of $18+$ to 24 months, $24+$ to 30 months, $30+$ to 36 months, $36+$ to 42 months and $42+$ to 48 months. For pilot study the stimuli were presented orally. Children were explained the task, they were instructed to listen to the full sentence and then to manipulate the toy as required. It was established that the children could follow the tasks appropriately. No further changes were made in the developed stimuli. The final test kit was then prepared in a bag containing the Chhota Bheem toy and the props to be used for the manipulation as per the stimulus sentences and is shown in the Figure 1 below.

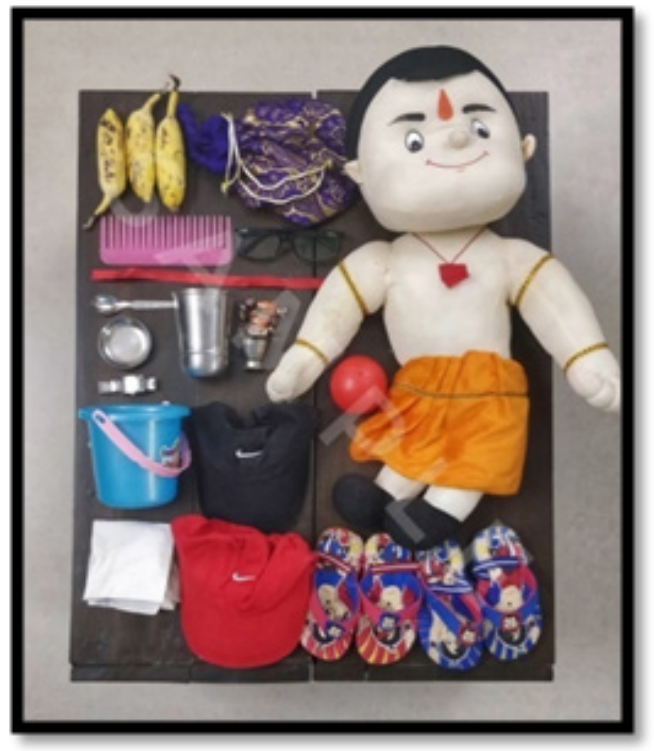

Figure I Testing Hindi pre-school children with normal hearing.

Participants: A total of 200 normal hearing children across the age range of 18 to 48 months, divided across five subgroups, were included as participants. Only children having normal speech developmental milestones as per Receptive Expressive Emergent Language Scale (REELS) ${ }^{31}$, normal otoscopic findings, no history of middle ear infection, a pass result on OAE screening in both ears and having Hindi speaking backgrounds at home were included. Children who had presence of middle ear infection, a refer result on the OAE screening in one or both ears or any associated impairments of any type such as cerebral palsy, mental sub-normality, visual impairment, developmental neuro-motor disabilities, attention deficit hyperactivity disorder and pervasive developmental disorders were excluded. Table 1 shows the details of the participants.

Test administration and scoring: Parents of all participants were given a brief overview of the test procedure. Written parental consent 
was mandatory prior to initiating any test procedure. Otoscopy was done using a hand-held Heine Otoscope in order to check for excessive cerumen or any other obvious abnormality of the external Table I Details of participants ear or tympanic membrane. TEOAE screening was done for both the ears using the Maico Eroscan 50.

\begin{tabular}{llll}
\hline Sub Group & Mean Age (Years) & Number & Gender \\
\hline I (18 to 24 months) & 1.766 & 40 & $20 \mathrm{M}, 20 \mathrm{~F}$ \\
2 (24 to 30 months) & 2.302 & 40 & $20 \mathrm{M}, 20 \mathrm{~F}$ \\
3 (30 to 36 months) & 2.742 & 40 & $20 \mathrm{M}, 20 \mathrm{~F}$ \\
4 (36 to 42 months) & 3.288 & 40 & $20 \mathrm{M}, 20 \mathrm{~F}$ \\
5 (42 to 48 months) & 3.76 & 40 & $20 \mathrm{M}, 20 \mathrm{~F}$ \\
\hline
\end{tabular}

The test was administered in a quiet one room setup, directly through the laptop connected to speakers. The practise list consisting of five sentences (long as well as short) was administered first. Each participant was instructed to listen to the sentences from the speaker and manipulate the toy as required. Each child was given a few minutes to get familiar with the toy and the prop and then the practice list was administered. Following the administration of the practise list and ensuring that the child has understood the procedure, lists 1 and 2 were administered. For half of the children list 1 was administered first followed by list 2; whereas for the other half, list 2 was administered first followed by list 1 . Each of the two lists contained 15 sentences each.

The participants' responses were recorded on the score sheet. There were 2 types of scores that were obtained - key word score and sentence score for each of the lists. Each of the sentences had a minimum of 2 and maximum of 4 key word scores. If all the key words were performed correctly then a sentence score of 1 was given, if not then a sentence score of 0 was given. A total key word score of 45 and sentence score of 15 could be obtained for the first list and a total key word score of 46 and sentence score of 15 could be obtained for the second list.

\section{Statistical analysis}

The sample consisted of 200 participants, which is a large sample. Considering this, parametric tests were applied. Descriptive statistics were obtained by calculating the means of scores obtained by the subjects in the five age groups. ANOVA was performed to test the statistical significance of the difference in the mean scores of the five groups. Following that post Hoc analysis using the Multiple Comparisons- Bonferroni test was done for pairwise comparisons. Pearson's Product-moment correlation was obtained to study the correlation between the two sentence lists of the developed test. Finally, item reliability was obtained using Cronbach's Alpha.

\section{Results}

Descriptive statistics were obtained by calculating the means of scores obtained by the subjects across the age range of 18 to 48 months for each of the two lists and also the key word scores of each of the lists.

\section{Keyword score for list I and list 2}

Table 2 shows the Mean, Standard Deviation (SD), Standard error, Minimum and Maximum values for the key word scores of sentence List 1 (L1) and key word scores of List 2 (L2) for each of the subgroups. The maximum attainable key word score for List 1 was 45 and for List 2 was 46 . The table thus depicts that the mean key word score for both List 1 and List 2 is minimum for sub-group 1, i.e. 18-24 months and maximum for sub-group 5, i.e. $42-48$ months. The mean scores thus increased as the age increased, indicating that the performance of normal hearing children improves with age.

The mean key word scores for sentence List 1 and List 2 for the different sub-groups are also depicted in Figure 2 There is a steep increase in scores from sub-group 1 to sub-group 2. Further ahead there is a constant increase as the age progresses. Ceiling scores have been obtained by four years of age. ANOVA indicated that the difference in mean scores of the 5 sub-groups is statistically significant $(p=.000)$. Bonferroni tests for multiple comparisons reveal that the mean scores of each sub-group differ significantly $(p=.000)$ from that of the other sub-groups.

\section{Sentence scores for sentence list I and list 2}

Table 3 shows the Mean, Standard Deviation (SD), Standard error, Minimum and Maximum values for the sentence scores for List 1 (L1) and List 2 (L2) for each of the subgroups. The maximum attainable sentence score for List 1 and List 2 was 15 . The table thus depicts that the mean sentence score for both lists was the minimum for sub-group 1, i.e. 18-24 months and maximum for sub-group 5, i.e. $42-48$ months. The mean scores thus increased as the age increased, indicating that for normal hearing children the performance improves with age. The mean sentence scores for sentence List 1 and List 2 for the different sub-groups are depicted in Figure 3. There is a constant increase as the age progresses. Ceiling scores have been obtained by four years of age. ANOVA indicated that the difference in mean scores of the 5 sub-groups is statistically significant $(p=.000)$. Bonferroni tests for multiple comparisons reveal that the mean scores of each sub-group differ significantly $(p=.000)$ from that of the other sub-groups. 
Table 2 Mean, SD, Standard Error, and range of keyword scores for List I (LI) and List 2 (L2) for different age groups

\begin{tabular}{|c|c|c|c|c|c|c|c|c|c|c|c|c|}
\hline \multirow[t]{2}{*}{$\begin{array}{l}\text { Age } \\
\text { Group }\end{array}$} & \multicolumn{2}{|l|}{$\mathrm{N}$} & \multicolumn{2}{|c|}{ Mean Score } & \multicolumn{2}{|c|}{ Std. Deviation } & \multicolumn{2}{|c|}{ Std. Error } & \multicolumn{2}{|c|}{$\begin{array}{l}\text { Minimum } \\
\text { score }\end{array}$} & \multicolumn{2}{|c|}{$\begin{array}{l}\text { Maximum } \\
\text { score }\end{array}$} \\
\hline & $\mathrm{LI}$ & L2 & $\mathrm{LI}$ & L2 & LI & L2 & LI & L2 & LI & L2 & $\mathrm{LI}$ & L2 \\
\hline $\begin{array}{l}18-24 \\
\text { months }\end{array}$ & 40 & 40 & 33.63 & 34.63 & 0.490 & .490 & 0.078 & .078 & 33 & 34 & 34 & 35 \\
\hline $\begin{array}{l}24-30 \\
\text { months }\end{array}$ & 40 & 40 & 37.90 & 37.83 & $0.84 I$ & .931 & 0.133 & .147 & 37 & 36 & 39 & 39 \\
\hline $\begin{array}{l}30-36 \\
\text { months }\end{array}$ & 40 & 40 & 40.48 & 41.13 & 0.506 & .822 & 0.080 & .130 & 40 & 40 & 41 & 42 \\
\hline $\begin{array}{l}36-42 \\
\text { months }\end{array}$ & 40 & 40 & 42.83 & 43.10 & 0.636 & 1.128 & 0.101 & .178 & 42 & 41 & 44 & 44 \\
\hline $\begin{array}{l}42-48 \\
\text { months }\end{array}$ & 40 & 40 & 44.68 & 45.68 & 0.474 & .474 & 0.075 & .075 & 44 & 45 & 45 & 46 \\
\hline Total & 200 & 200 & 39.90 & 40.47 & 3.930 & 3.908 & 0.278 & .281 & 33 & 34 & 45 & 46 \\
\hline
\end{tabular}

Table 3 Mean, SD, Standard Error, and range of sentence scores for List I (LI) and List 2 (L2) for different age groups

\begin{tabular}{|c|c|c|c|c|c|c|c|c|c|c|c|c|}
\hline \multirow[t]{2}{*}{$\begin{array}{l}\text { Age } \\
\text { Group }\end{array}$} & \multicolumn{2}{|l|}{$\mathrm{N}$} & \multicolumn{2}{|c|}{ Mean Score } & \multicolumn{2}{|c|}{ Std. Deviation } & \multicolumn{2}{|c|}{ Std. Error } & \multicolumn{2}{|c|}{$\begin{array}{l}\text { Minimum } \\
\text { score }\end{array}$} & \multicolumn{2}{|c|}{ Maximum score } \\
\hline & LI & L2 & LI & L2 & LI & L2 & LI & L2 & LI & L2 & LI & L2 \\
\hline $\begin{array}{l}\text { I8-24 } \\
\text { months }\end{array}$ & 40 & 40 & 8.63 & 8.63 & .490 & .490 & .078 & .078 & 8 & 8 & 9 & 9 \\
\hline $\begin{array}{l}24-30 \\
\text { months }\end{array}$ & 40 & 40 & 9.50 & 9.43 & .506 & .501 & .080 & .079 & 9 & 9 & 10 & 10 \\
\hline $\begin{array}{l}30-36 \\
\text { months }\end{array}$ & 40 & 40 & I I.43 & 11.43 & .501 & .501 & .079 & .079 & 11 & II & 12 & 12 \\
\hline $\begin{array}{l}36-42 \\
\text { months }\end{array}$ & 40 & 40 & 12.88 & 12.85 & .563 & .533 & .089 & .084 & 12 & 12 & 14 & 14 \\
\hline $\begin{array}{l}42-48 \\
\text { months }\end{array}$ & 40 & 40 & 14.68 & 14.68 & .474 & .474 & .075 & .075 & 14 & 14 & 15 & 15 \\
\hline Total & 200 & 200 & I I.42 & 11.40 & 2.263 & $2.27 \mathrm{I}$ & .160 & .161 & 8 & 8 & 15 & 15 \\
\hline
\end{tabular}
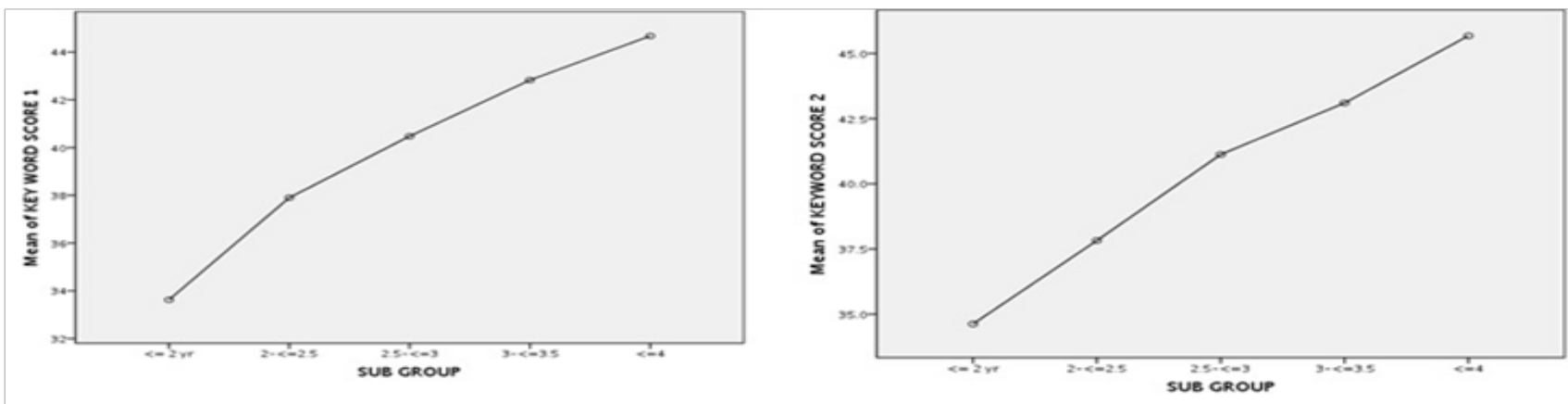

Figure 2 Mean key word scores for List I (LI) and List 2 (L2) for different age groups. 

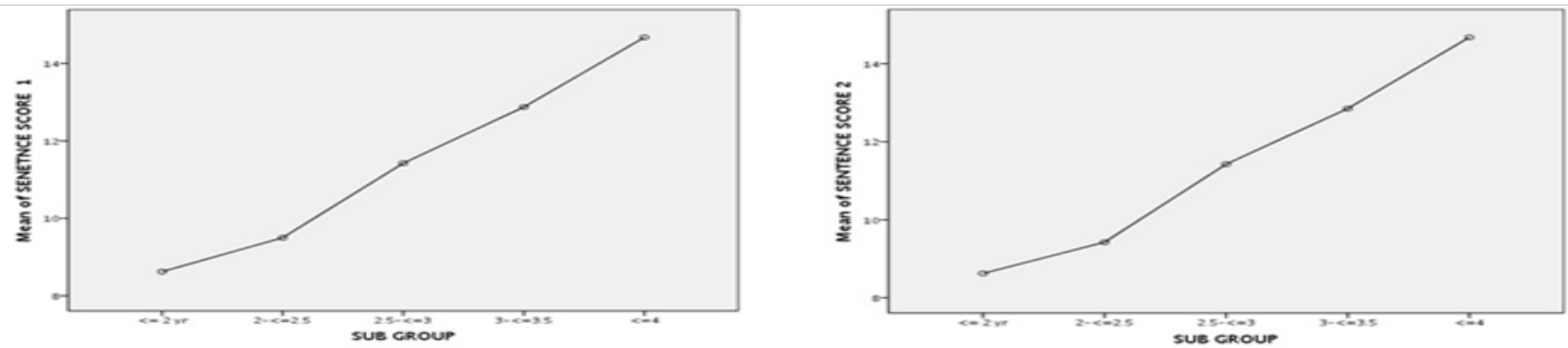

Figure 3 Mean sentence scores for List I (LI) and List 2 (L2) for different age groups.

\section{Correlation between the two lists}

In order to measure the strength of association between the key word scores and sentence scores of List 1 and key word scores and sentence scores of List 2, Karl Pearson's product moment correlation was computed. Obtained r-values of .987 and .998 for key word and sentence scores respectively, demonstrate a high positive correlation between the scores obtained on the two lists. The high positive correlation indicates that the children who are getting high scores on List 1 will also have high scores on List 2.

\section{Item reliability}

The obtained Cronbach's Alpha values were .894 for key words List 1, 0.886 for key words List 2, .852 for sentence List 1 and .853 for sentence List 2, all indicative of high item reliability.

\section{Discussion}

Robbins and $\mathrm{Kirk}^{32}$ while assessing paediatric cochlear implant users suggested the use of SCIPS, PSI, GAEL-P, MAIS and Mr. Potato Head task for assessment of speech perception skills in preschool children. Zeng et $\mathrm{al}^{33}$ used a recorded administration procedure during the development of the Mandarin Early Speech Perception test for 2-5-year-old developmentally normal children. It was seen that recorded test could also be used with children who successfully wore hearing aids and cochlear implants. Oyer and Doudna ${ }^{34}$ commented that most open set tests are not appropriate for young paediatric populations because open set tests carry higher perceptual, linguistic and memory demands than closed set tests. Hence a modified open set sentence test like the one developed in the study is a good option to test speech perception skills in young children.

Newton, Chiat and Dankovicova ${ }^{35}$ assessed speech discrimination in 50 normally developing children, divided into five age groups of 2-2.11, 3-3.11, 4-4.11, 5-5.11, 6-6.11 years using a set of stimuli which included 5 minimal word pairs with different place of articulation of the initial consonant, 5 pairs with different manner of articulation of the initial consonant, 5 pairs differing by voicing of the initial consonant, 5 pairs differing by voicing, place and manner of the initial consonant and 10 minimal pairs differing by vowel quality. A picture pointing task was used as the response mode. Results revealed that all the typically developing children did very well on the picture pointing task, though their accuracy still increased with age, such that 2 -year olds got a percentage score of $86 \%$ while the 5 - and 6 -year olds obtained ceiling scores. This finding is replicated in the present study where performance is improving as a function of age. Thus, as age and hearing experience advances, the receptive language skills improve. Child understands tasks better and performs better with advancement in age and hearing experience. Similar results are obtained in present study, where perception skills improve as age increases.

Jerger, Lewis, Hawkins and Jerger in $1980^{36}$ developed the Paediatric Speech Intelligibility sentence material for children between the ages of 3 to 6 years. They incorporated the consistent differences in vocabulary and receptive language function that were characterized by the children in that age group. To represent the different sentence patterns produced by the children sampled, two 10-sentence lists were formed using two difference syntactic constructions. Format 1 consists of the sentence construction "article noun/verbing/article noun" and sentences are preceded by the carrier phrase, "show me," such as "Show me a rabbit reading a book." For Format 1 materials, excluding the carrier phrase, the average sentence length was kept as 7 syllables. Format 2 consists of the sentence construction "article noun/auxiliary verb-in/ auxiliary noun," and sentence materials are not preceded by carrier phrase (e.g., "A bear is eating a sandwich."). For Format 2 materials, average sentence length was kept as 8 syllables. For Format 1 sentences, the correlation coefficient between the two lists was 0.75 ; for Format 2 sentences, the correlation coefficient between the two lists was 0.70 indicating no significant inter- list differences for either Format 1 or 2 . Such studies as well as results from the present study indicate the importance of having equivalent alternate forms of a test that can be used interchangeably.

\section{Conclusion}

There was a significant difference in the scores between the 5 age groups of children with normal hearing. There was an increase in scores as age increased, thus indicating improved performance with increasing age. There was also no significant difference in the performance on List 1 and List 2 for the participants indicating that the two lists were equivalent. Item reliability was also observed to be high. In conclusion, the developed Modified Open Set Sentence Test can be successfully used to assess speech perception skills in young Hindi children with normal hearing. This test has a potential to be a useful clinical tool when used as a part of a test battery and play a role in monitoring the progress of an intervention program. Given that more and more young children are being implanted, appropriate speech perception testing procedures are necessary for cochlear implant candidacy assessment as well as for measuring outcomes in the immediate period after implantation. This test provides such a tool for the young population.

\section{Implications}

a) The study has developed a modified open set sentence test of 
speech perception for children with normal hearing in the age range of one and a half to four years. The developed test can be used in clinical assessment of speech perception of children in this age group. The test can be useful in other areas like candidacy assessment for cochlear implant surgery, pre and post-operative CI assessment, and planning of therapy goals.

b) The results of the study have provided insight into the performance of children with normal hearing. The performance can reflect the communication ability in everyday situation.

c) The test was administered without an audiometer directly using speakers which permits wider use of the test in routine clinical practice.

\section{Acknowledgments}

Authors would like to acknowledge Director, AYJNISHD, Mumbai; families of all children who participated in the study; the playgroups who extended help in recruiting the participants; and the parents and professionals who helped in test development.

\section{Conflicts of interest}

The authors declare no conflicts of interest.

\section{References}

1. Boothroyd A. Speech Perception and Sensorineural Hearing Loss. In M Ross \& T. G. Giolas (Eds.). Auditory Management of Hearing-Impaired Children: Principles and Prerequisites for Intervention. (Perspectives in Audiology Series) (118-142), Baltimore: Park University Press; 1978.

2. Ryalls J. A Basic Introduction to Speech Perception. London: Singular Publishing Grp; 1996.

3. Giolas TG, Epstein A. Comparative intelligibility of word lists and continuous discourse. J Speech Hear Res. 1963;6:349-359.

4. Epstein A. Speech audiometry. Otolaryngologic Clinics of North America, 1978;11:667-676.

5. Davis JM. Performance of young hearing-impaired children on a test of basic concepts. Journal of Speech Hearing Research. 1994;17(3):342-351.

6. Silverman SR, Hirsh IJ. Problems related to the use of speech in clinical audiometry. Ann Otol Rhinol Laryngol. 1955;64(4): 1234-1245.

7. Boothroyd A. Developments in speech audiometry. International Audiology. 1968;7:363-368.

8. Jerger J, Speaks C, Trammell JL. A new approach to speech audiometry. $J$ Speech Hear Disord. 1968;33(4):318-28.

9. Martin FN, Jansen RM. Speech reception thresholds using conventional vs high-frequency spondees in normals and in subjects with marked highfrequency sensorineural loss. J Aud Res. 1985;25(2):133-142.

10. Nikolopoulos TP, O Donoghue GM, Archbold S. Age at implantation its importance in pediatric cochlear implantation. Laryngoscope. 1999;109(4):595-599.

11. Lehnhardt E, Langenbeck B. Praktische Audiometric: Lehrbuch und synoptischer Atlas. Georg Thieme Verlag, 1978.

12. Martin FN, Armstrong TW, Champlin CA. A survey of audiological practices in the United States. Am J Audiol. 1994;3(2):20-6.

13. Martin FN, Forbis NK. The present status of audiometric practice: A follow- up study. ASHA. 1978;20(7):531-541.

14. Martin FN, Pennington CD. Current trends in audiometric practices. ASHA. 1971;13(11):671-677.
15. Kalikow DN, Stevens KN, Elliott LL. Development of a test of speech intelligibility in noise using sentence materials with controlled word predictability. J Acoust Soc Am. 1977;61(5):1337-1351.

16. Cox RM, Alexander GC, Gilmore C. Development of the Connected Speech Test (CST). Ear Hear. 1987;8(5):119s.

17. Erber NP. Use of the auditory numbers test to evaluate speech perception abilities of hearing-impaired children. $J$ Speech Hear Disord. 1980;45(4):527-532.

18. Finitzo Hieber T, Matkin N, Cherow Skalka E, et al. Sound effects recognition test (SERT). St Louis, MO: Auditec; 1977.

19. Black JW. Multiple-choice intelligibility tests. $J$ Speech Hear Disord. 1957;22(2):213-235.

20. Cheng AK, Grant GD, Niparko JK. Meta-analysis of pediatric cochlear implant literature. Ann Otol Rhinol Laryngol Suppl. 1999;177:124-8.

21. https://govinfo.me/sruthitharangam-scheme/

22. http://disabilityaffairs.gov.in/content/page/adip-scheme.php

23. Zimmerman phillips S, Robbins AM, Osberger MJ. Assessing cochlear implant benefit in very young children. Ann Otol Rhinol Laryngol Suppl. 2000;109(12):42-43.

24. Robbins AM, Renshaw JJ, Berry SW. Evaluation of meaningful auditory integration in profoundly hearing impaired children. Am J Otol. 1991;12(Suppl):114-50.

25. Ching TYC, Hill M. The Parents' Evaluation of Aural/Oral Performance of Children (PEACH) Scale: Normative Data. J Am Acad Audiol. 2007;18(3):220-235.

26. Moog JS, Geers AE. (1990). Early Speech Perception Test for Profoundly Hearing Impaired Children. St. Louis: Central Institute for the Deaf 1990. Cited in Kirk KI, Diefendorf AO, Pisoni DB, Robbins AM. Assessing Speech Perception in Children. In Mendel L, Danhauer JL. Audiologic Evaluation and Management and Speech Perception Assessment. San Diego: Singular Publishing Group 1997. p. 101-132.

27. Ross M, Lerman J. A Picture Identification Test for Hearing Impaired Children. J Speech Hear Res. 1979;13(1):44-53.

28. Ling D, Ling AP. Aural Habilitation: The foundations of verbal learning. Washington DC: AG Bell Association of Deaf, 1978.

29. Census of India. Abstract of speakers' strength of languages and mother tongues. 2001.

30. Robbins AM. Mr. Potato Head task. Indianapolis, IN: Indiana University School of Medicine; 1994.

31. Bzoch K, League R. Receptive-Expressive Emergent Language (REEL) scale. Austin, TX: Pro-Ed, 1971

32. Robbins A, Kirk K. Speech Perception Assessment and Performance in Pediatric Cochlear Implant Users. Seminars in Hearing 1996;17(4):353369.

33. Zeng FG, Galvin III JJ. Amplitude mapping and phoneme recognition in cochlear implant listeners. Ear Hear. 1999;20(1):60-74.

34. Oyer W, Doudna M. Structural analysis of word responses made by hard of hearing subjects on Discrimination test. AMA Arch Otolaryngol. 1959;70:357-364.

35. Newton C, Chiat S, Dankovicova J. Evaluation of a Novel Technique for Assessing Speech Discrimination. A research project based at university College London and supported by a grant from the Economic and Social Research Council. (Award number: RES-000-22-0748). 2014.

36. Jerger S, Lewis S, Hawkins J, et al. Pediatric speech intelligibility test. Generation of test materials. Int $J$ Pediatr Otorhinolaryngol. $1980 ; 2(3): 217-230$ 